\title{
Multi-wavelength analysis of the importance 3B/M7.1 flare on September 231998
}

\author{
E.S. Kulagin ${ }^{1}$, P.G. Papushev ${ }^{2}$, S.A. Chuprakov ${ }^{2}$ \\ ${ }^{1}$ Central (Pulkovo) Observatory of the Russian Academy of Sciences, email: galkin@gao.spb.ru \\ ${ }^{2}$ Institute for Solar-Terrestrial Physics of RAS (Siberian branch), email: papushev@iszf.irk.ru
}

\begin{abstract}
The coincidence was observed between the places with weak (probably inverse) photospheric longitudinal magnetic fields in the big spot of the AR NOAA 8340 prior to the start of the flare and a common base of the giant arches (loops) and centers of emission visible in the HeI $10830 \AA$ line core during the flare. A number of observational facts compel us to make a supposition that the flare was the consequence of an injection of plasma from the places with marked peculiarity of the photospheric magnetic field into the expanding magnetic arcade.
\end{abstract}

We have investigated the importance 3B/M7.1 flare that have occurred near the center of the solar disk on September 231998 in the active region NOAA 8340 with dominant big spot at the heliographic coordinates E09, N19. In the consent with Altyntsev et al. (2002) we can distinguish four stages in the flare evolution: from 6:44 till 6:54 — initial stage, from 6:54 till 6:58 - impulsive stage, from 6:58 till 7:13 - main stage, and after 7:13 - decay stage (hereafter universal time). A series of another articles (Kotrč et al. 1999; Altyntsev et al. 2002) is dedicated to this powerful flare.

Images of the flare in the core of the HeI $10830 \AA$ line were obtained at the Pulkovo Observatory using the narrow-band tunable filter (Kulagin 1999). FWHM of the filter was $0.24 \AA$. The filtergrams were recorded with a SBIG ST-6 CCD camera. Unfortunately, the observation in the helium line has begun at $6: 57,13 \mathrm{~m}$ after the start of the flare. But using the 2D-spectroscopy technique and specially designed computer program, we were lucky to receive the small maps of the longitudinal magnetic fields and Doppler velocities within the SiI $10827 \AA$ line $46 \mathrm{~m}$ before the start of the flare.

In the Figure above, four images of the flare are presented. The size of these images is $130^{\prime \prime} \times 130^{\prime \prime}$. It is determined by the size of our small magnetogram. North is approximately at the top, east is at the left. Below each image, an ordinal number and time are given. The first image was obtained in continuum. The second one is the map of the longitudinal magnetic fields $46 \mathrm{~m}$ prior to the flare. Here positive magnetic polarity is shown in white, the negative one - in black. The areas of weak magnetic field look grey. In the region of umbrae and penumbra of the big spot the strong field of positive polarity up to 1500 Gs was observed. But four small places with weak field, surrounded by the strong field, which are situated near the umbra-penumbra boundaries of the big spot, are seen. These places are visible as four grey blobs on white background. A longitudinal magnetic field of positive polarity less than $200 \mathrm{Gs}$ and gradient of up to $0.4 \mathrm{Gs} \mathrm{km}^{-1}$ are observed here. Taking into consideration the low spatial resolution of the map (about $\left.3^{\prime \prime}\right)$, it is possible to suspect the opposite magnetic polarity and lager gradient in these places. The third image is the filtergram obtained in the core of the HeI $10830 \AA$ line at the impulsive stage of the flare. The three giant absorption arches are visible here. The fourth image is the simultaneous filtergram in the CIV $1550 \AA$ line from "Trace". The common base of the absorption arches visible in helium is indicated by arrow in all images. 


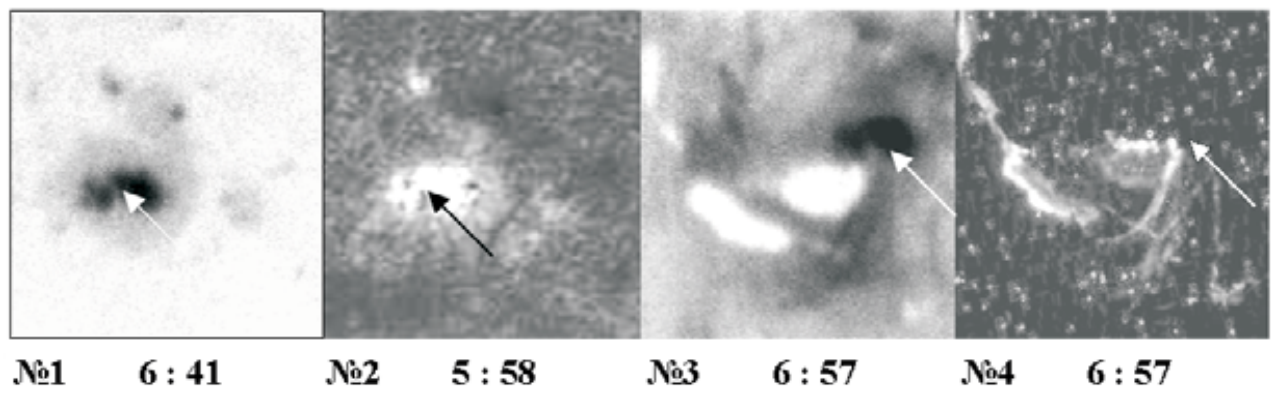

We consider our main observational results as follows:

(1) four places with weak (probably inverse) photospheric longitudinal magnetic fields with large gradient were observed $46 \mathrm{~m}$ prior to the start of the flare near the umbrapenumbra boundaries of the big spot. All these places have displayed themselves as a common base of the giant arches, centers of emission in the helium line, or as a center of outflow prior to the flare in the silicon line (in the rightmost one of them);

(2) the arches connected the big spot to the principal emission zone moving eastwards;

(3) the arch structures were better visible in the impulsive stage of the flare in the helium line than in the carbon line. It tells us, apparently, of the low temperature of arches in the impulsive stage;

(4) prior to the main stage of the flare, the leading edge of the principal emission zone was steep, while after the main stage the back edge became steep. The effect of turn-on and turn-off a fountain was clearly observed in the helium line;

(5) the maps of the longitudinal magnetic field of the Kitt Peak observatory for the neighboring dates showed that, most probably, amplification of the magnetic field of the big spot took place in the course of the flare.

On the base of these observational data we make a supposition that, in the course of development of the flare, the injection of plasma into the expanding arcade took place from the three places of the photosphere (except the rightmost one mentioned above, where outflow was observed prior to the flare). The injection can be a consequence of the magnetic flux cancellation in the photospheric or sub-photospheric level. Such events were observed with the vector magnetograph (Wang Jingxiu and Shi Zhongxian 1993).

\section{Acknowledgements}

The authors thank the "Trace" space observatory team for the high-quality images of the flare. NSO/Kitt Peak data used here are produced cooperatively by NSF/NOAO, NASA/GSFC, and NOAA/SEL.

\section{References}

Altyntsev, A.T., Sych, R.A., Grechnev, V.V., Meshalkina, N.S., and Rudenko, G.V. 2002 Solar Phys. 206, p. 155.

Kotrč, P., Karlický. M., Kupryakov,Yu.A., Kaltman, T.I., Kašparova, J., Rompolt, B. In Proc. 9-th European Meeating on Solar Physics, "Magnetic Fields and Solar Processes", Florence, Italy, 12-18 September 1999 (ESA SP-448, December 1999), p. 841.

Kulagin, E. S. 1999 Solar Phys. 188, p. 81.

Wang Jingxiu and Shi Zhongxian 1993 Solar Phys. 143, p. 119. 